Gut, 1965, 6, 372

\title{
Radiological diagnosis of gastritis
}

\author{
W. B. JAMES, A. G. MELROSE, J. W. DAVIDSON, AND R. I. RUSSELL \\ From the Departments of Radiology and Medicine, Southern General Hospital, \\ Glasgow
}

EDITORIAL SYNOPSIS Considerable success has been achieved in the radiological diagnosis of gastric atrophy.

The majority of physicians believe that there is no diagnostic symptom complex by which chronic gastritis can be recognized and that it is difficult or impossible to diagnose the disease clinically. The advent of gastric biopsy permitted an agreed histological classification of diffuse mucosal abnormalities, and has allowed the results obtained by other methods to be subjected to comparison with the histological findings. It has become evident that clinical and gastroscopic diagnoses of gastritis bear little relation to the mucosal changes seen on microscopy. Radiological descriptions of supposed signs of chronic gastritis, such as 'thickened rugae', and 'enlarged folds', were based to a large extent on gastroscopic observations, and they too have fallen into disrepute. Joske, Finckh, and Wood (1955) compared the radiological and histological findings in patients with various types of gastritis and could find no correlation. It seemed therefore that radiology had little to contribute to the study of diffuse gastric mucosal lesions. In 1956, however, Badenoch, Kemp, and Richards were able, in a retrospective study, to make a correct diagnosis on radiological grounds of either normality or gastric atrophy in 28 of 35 patients. Their observations have since been confirmed by Laws and Pitman (1960) and by Joske and Vaughan (1962), and criteria for a radiological diagnosis of gastric atrophy have been established. By contrast, no correlation has been demonstrated between the radiological and histological findings in superficial gastritis (Henning, Heinkel, and Frik, 1960; Joske and Vaughan, 1962; Berlin, 1962), and this lesion cannot be distinguished from normal mucosa by radiological methods.

There is the possibility therefore of differentiating radiologically between normal mucosa, including superficial gastritis, on the one hand, and the atrophic mucosa on the other. In this study we have investigated the accuracy of the radiological assessment of diffuse lesions of gastric mucosa by correlating the opinions of two radiologists on radio- graphs of the stomach taken at routine barium meal examination with the histological findings from gastric biopsy specimens.

\section{MATERIAL AND METHODS}

Gastric biopsy was carried out on 45 patients, using Wood's flexible gastric biopsy instrument. All the patients had previously suffered from duodenal ulcer. As recommended by Bockus (1963a and b), whenever possible more than one biopsy was taken from each patient, and in the group examined the average number of specimens per patient was two. The assumption has been made that the biopsy material is representative of the overall state of the mucosa in the body of the stomach, and we believe this assumption to be justified, in view of the observations of Williams, Edwards, Lewis, and Coghill (1957), who noted agreement in approximately $90 \%$ of samples taken at the same time, irrespective of the site of the various samples.

Each section was stained with haematoxylin and eosin, a trichromic stain, and a mucin stain. If two or more specimens were obtained, it was known that they came from the same patient. Three stains were performed on each biopsy, and at least two sections were stained by each method; on average six slides per biopsy were examined. In order to minimize observer error the slides were reviewed on two subsequent occasions before analysis was undertaken.

Histological CLASSIFICATION The histological classification is that of Bock, Richards, and Witts (1963), namely:

1 Normal.

2 Superficial gastritis There was inflammatory cell infiltration of the mucosa, often with distortion of the superficial layers but no evidence of atrophy of the specific cells.

3 Atrophic gastritis There was inflammatory cell infiltration of the mucosa with variable but not complete atrophy of the specific cells. Islands of intestinal metaplasia were sometimes present.

4 Gastric atrophy Atrophy of the specific cells was severe or complete, intestinal metaplasia was often 
conspicuous, and there was little or no inflammatory cell infiltration of the mucosa.

Twelve patients with Addisonian pernicious anaemia were added to the group of 45 patients who had had a biopsy, bringing the total number of subjects to 57 . The 12 patients with pernicious anaemia were presumed for the purpose of this investigation to have gastric atrophy and biopsy was not performed. In each case a diagnosis of Addisonian pernicious anaemia had been established beyond doubt by demonstration of a typical peripheral blood picture, megaloblastic bone marrow, histaminefast achlorhydria, a subnormal serum $B_{12}$ level, and complete correction of the anaemia by the administration of vitamin $\mathrm{B}_{12}$ alone.

Radiographs of the stomach obtained at routine barium meal examination in these 57 patients were assessed independently by two radiologists. In all cases, supine and prone films with the mucosa coated with barium, and supine and erect films with the stomach full of barium were available.

Each radiologist recorded whether the mucosa was normal or abnormal, and if abnormal, whether the changes should be classified as gastric atrophy or atrophic gastritis. At the time of assessment the radiologist did not know the diagnosis made by his colleague, nor did he know the result of the gastric biopsy.

RADIOLOGICAL CRITERIA The following radiological criteria of gastric atrophy were used in assessment: (1) A 'tube'-shaped stomach; (2) absent or diminished rugal markings along the greater curve; (3) a 'bald' fundus; and (4) very fine mucosal folds in the body and fundus. In practice, the first two of these criteria were found to be most useful.

When such changes were gross, a diagnosis of 'gastric atrophy' was made. When they were considered less evident, but nevertheless present, the case was classified as 'atrophic' gastritis. This distinction is quite arbitrary, and subject to considerable variation in individual interpretation. The reason for attempting to make the distinction will be discussed later.

\section{RESULTS}

These are shown in Tables I-III. The data have been grouped into normal, i.e., normal and superficial gastritis, and abnormal, i.e., atrophic gastritis

\section{TABLE I}

DIAGNOSIS MADE BY RADIOLOGIST 1 CONTRASTED WITH HISTOLOGICAL FINDINGS

Histological Diagnosis Radiological Diagnosis

$$
\begin{array}{llll}
\text { Normal } & \text { Super- Atrophic Gastric Total } \\
& \text { ficial } & \text { Gastritis Atrophy }
\end{array}
$$

\begin{tabular}{lrlrlr}
\hline Normal & 4 & 0 & 0 & 0 & 4 \\
Superficial gastritis & 17 & 0 & 4 & 0 & 21 \\
Atrophic gastritis & 16 & 0 & 3 & 1 & 20 \\
Gastric atrophy & 1 & 0 & 3 & 8 & 12 \\
& 38 & 0 & 10 & 9 & 57
\end{tabular}

TABLE II

DIAGNOSIS MADE BY RADIOLOGIST 2 CONTRASTED WITH HISTOLOGICAL FINDINGS

Histological Diagnosis Radiological Diagnosis

\begin{tabular}{rlrrr}
\hline Normal & $\begin{array}{l}\text { Super- } \\
\text { ficial } \\
\text { Gastritis }\end{array}$ & $\begin{array}{l}\text { Atrophic Gastric } \\
\text { Gastritis }\end{array}$ & Ttrophy \\
& & & \\
\hline 4 & 0 & 0 & 0 & 4 \\
18 & 0 & 2 & 1 & 21 \\
16 & 0 & 2 & 2 & 20 \\
2 & 0 & 3 & 7 & 12 \\
40 & 0 & 7 & 10 & 57
\end{tabular}

TABLE III

DIAGNOSIS OF RADIOLOGISTS 1 AND 2 COMPARED

Radiologist 1

Radiologist 2

Normal Atrophic Gastric Total Gastritis Agrophy

Normal

Atrophic gastritis

Gastric atrophy

$\begin{array}{rrrr}36 & 2 & 0 & 38 \\ 4 & 3 & 3 & 10 \\ 0 & 2 & 7 & 9 \\ 40 & 7 & 10 & 57\end{array}$

and gastric atrophy, and the two groups compared.

The validity of the radiological diagnosis as between atrophic gastritis and gastric atrophy has been calculated for each radiologist (Tables I and II), and finally, the accuracy of radiologist 1 as compared with radiologist 2 , in differentiating normal from abnormal, and atrophic gastritis from gastric atrophy has been defined (Table III).

There was better than chance agreement between radiological and histological diagnosis in $63 \%$ of cases $\left(x^{2}=4.68, \mathrm{p}<0.05\right.$ probably statistically significant) assessed by radiologist 1 (Table I). Where a positive radiological diagnosis of normality had been made, this was correct in $55 \%$ of cases. Where a positive radiological report of abnormality had been made this was correct in $79 \%$ of cases. Where a positive radiological report of either atrophic gastritis or gastric atrophy had been made this was correct in $73 \%$ of cases.

Agreement was present between radiological and histological diagnosis in $63 \%$ of cases $\left(\chi^{2}=5 \cdot 35\right.$, $\mathrm{p}<0.05$, probably statistically significant) assessed by radiologist 2 (Table II). Where a positive radiological report of normality had been made, this was correct in $55 \%$ of cases. Where the report showed definite abnormality, this was in fact present in $82 \%$ of cases. A positive radiological report of atrophic gastritis or gastric atrophy was correct in $64 \%$ of cases.

Comparing the reliability of radiologist 1 against radiologist 2 (Table III), there was agreement that the mucosa was either normal or abnormal in $89 \%$ of cases $\left(x^{2}=29.4, \mathrm{p}<0.001\right.$ statistically significant). When a positive radiological diagnosis of 
atrophic gastritis or gastric atrophy had been made the radiologists were in agreement in $67 \%$ of cases $\left(x^{2}=0 \cdot 31\right.$, not statistically significant).

\section{DISCUSSION}

Contrary to the opinions of Glass (1957) and Schinz, Baensch, Friedl, and Uehlinger (1954) that the radiological diagnosis of gastric atrophy is unsatisfactory, we agree with Badenoch et al. (1956), with Laws and Pitman (1960), and with Joske and Vaughan (1962) that there are characteristic radiological appearances associated with severe diffuse gastric mucosal atrophy, and we have shown that there is a close measure of agreement between radiologists on the recognition of these signs. Faber (1927) considers that gastric atrophy begins as a superficial gastritis, and subsequently passes through a stage of atrophic gastritis. Magnus (1958) and Davidson and Markson (1955) also believe that atrophic gastritis and gastric atrophy represent different stages of the same pathological process. It appears then that the mucosal lesions in pernicious anaemia may be less severe than complete gastric atrophy, and may consist of a severe atrophic gastritis. The assumption that atrophic gastritis differs from gastric atrophy only in degree was the reason for our attempt to differentiate the two on radiological grounds. We found that our overall accuracy in diagnosing atrophic gastritis was poor, as was agreement between the two radiologists on this topic. Our experience contrasts with that of Bock, Kemp, and Richards (1963), who were able to diagnose 'gastric atrophy' radiologically, in seven out of nine patients who had a histological diagnosis of atrophic gastritis.

It should be recognized that when the radiologist refers to 'mucosal pattern' in barium meal studies, he is describing the mucosal relief pattern, formed by barium outlining the raised folds of mucosa. It is likely that these folds are due to contractions of the underlying muscularis mucosae (Taft, 1959). Magnus (1958) has drawn attention to atrophy of the muscle coat of the stomach in pernicious anaemia, and we agree with Joske and Vaughan (1962) that such changes could form a pathological basis for the appearances seen radiologically in gastric atrophy. This would also explain why histological atrophic gastritis without significant muscle coat atrophy can produce a normal radiological appearance. Joske and Vaughan (1962), who divided their cases of atrophic gastritis into moderate and severe groups histologically, showed that the frequency of correct radiological diagnoses of atrophy in atrophic gastritis increased with the severity of the histological lesion. In those cases with histological gastric atrophy there was almost complete agreement between biopsy and radiological diagnosis. In short, a radiological diagnosis of gastric atrophy indicates that biopsy will show gastric atrophy or severe atrophic gastritis in the great majority of cases.

It is extremely unlikely that the suspicion that pernicious anaemia is present will first arise following barium meal examination, though in our practice we comment on the presence of radiological signs of gastric atrophy when they are found. Nevertheless, it should be remembered that gastric atrophy may precede the development of both pernicious anaemia and subacute combined degeneration (Robertson, Wood, and Joske, 1955). The radiographic finding of gastric atrophy should therefore be drawn to the attention of the clinician. Conversely, if in a patient with a megaloblastic anaemia there is no evidence of radiological gastric atrophy, it is likely that the patient is not suffering from Addisonian pernicious anaemia.

The present investigation is the first statistical examination of the accuracy of the radiological method in the diagnosis of gastric atrophy and atrophic gastritis. Important points emerging are that radiological differentiation of abnormal from normal mucosa was only fair, $63 \%$ for each radiologist, but when a positive radiological report of abnormality could be made, accuracy rose to $79 \%$ and $82 \%$. Radiological differentiation of atrophic gastritis from gastric atrophy was also only fair, $73 \%$ and $64 \%$. There was a close measure of agree. ment between radiologists in separating the normal from the abnormal in $89 \%$ of cases, but agreement was much less close in separating cases of atrophic gastritis from gastric atrophy (67\% of cases). Differentiation between atrophic gastritis and gastric atrophy was not accurate enough to be of value, and a correct diagnosis could often occur by chance. In clinical practice, however, such differentiation is not important, it being sufficient to know that the mucosa shows evidence of atrophy, whether or not it is complete. This study confirms that any marked degree of atrophic change in the gastric mucosa will be revealed by the radiological method in a high proportion of cases.

\section{SUMMARY}

Barium meal examination was carried out in 45 patients who had had gastric biopsy, and on 12 patients with pernicious anaemia who were presumed to have gastric atrophy. An attempt was made, independently, by two radiologists (1) to state whether the stomach appeared to be normal, or whether there was evidence of mucosal atrophy, and (2) if evidence of atrophy was considered to be present, 
whether this represented complete gastric atrophy or atrophic gastritis.

The diagnostic accuracy of each radiologist was investigated, and their findings compared. The results were subjected to statistical analysis. When a radiological report of abnormality indicative of any degree of mucosal atrophy could be made, this was correct in four out of five cases and agreement existed between radiologists in nine out of 10 cases. It was not possible to differentiate accurately between atrophic gastritis and gastric atrophy.

We wish to thank Dr. A Dick, who reported on the. histology of the gastric biopsy specimens, and Dr. J. K. Davidson for help with the manuscript. Mr. Ralph McGuire kindly carried out the statistical analysis of the results.

\section{REFERENCES}

Badenoch, J., Kemp, F. H., and Richards, W. C. D. (1956). The radiological diagnosis of gastric atrophy. Correlation between radiographic studies and histological appearances. Gastroenterologia (Basel), 85, 216-223.

Berlin, L. (1962). Gastritis: a medical dilemma. Amer. J. Roentgenol., 88, 627-636.

Bock, O. A. A., Kemp, F. H., and Richards, W. C. D. (1963). The radiological diagnosis of gastric atrophy. Brit. J. Radiol., 36, 578-582.
—-, Richards, W. C. D., and Witts, L. J. (1963). The relationship between acid secretion after augmented histamine stimulation and the histology of gastric mucosa. Gut, 4, 112-114.

Bockus, H. L. (1963a). Gastroenterology, 2nd ed., vol. 1, p. 384. Saunders, Philadelphia.

- (1963b). Ibid., vol. 1, pp. 415-416. Saunders, Philadelphia.

Davidson, W. M. B., and Markson, J. L. (1955). The gastric mucosa in iron deficiency anaemia. Lancet, 2, 639-643.

Faber, K. (1927). The etiology and pathogenesis of achylia gastrica. In Lectures on Internal Medicine, pp. 1-43. Hoeber, New York.

Glass, G. B. J. (1957). Gastric atrophy. Its clinical significance and methods of detection. Amer. J. dig. Dis., 2, 709-732.

Henning, N., Heinkel, K., and Frik, W. (1960). Gastric mucosal pattern. A comparison of the radiological pattern of the gastric mucosal folds with the histological findings by biopsy. Germ. med. Mth., 5, 408-413.

Joske, R. A., and Vaughan, B. F. (1962). The radiologic findings in histologically verified atrophic gastritis and gastric atrophy. Gastroenterology, 42, 7-15.

—, Finckh, E. S., and Wood, I. J. (1955). Gastric biopsy. A study of 1,000 consecutive successful gastric biopsies. Quart. J.Med. 24, 269-294.

Laws, J. W., and Pitman, R. G. (1960). Malabsorption syndromes. IV. The radiological features of pernicious anaemia. Brit. $J$ Radiol., 33, 229-237.

Magnus, H. A. (1958). A re-assessment of the gastric lesion in pernicious anaemia. J. clin. Path., 11, 289-295.

Robertson, E. G., Wood, I. J., and Joske, R. A. (1955). Gastric atrophy with subsequent pernicious anaemia and pronounced subacute combined degeneration of the cord. Lancet, 2, 69-71.

Schinz, H. R., Baensch, W. E., Friedl, E., and Uehlinger, E. (1954). Roentgen-Diagnostics, vol. 4, p. 3885. Grune and Stratton, New York.

Taft, L. I. (1959). Aspects of chronic gastritis. Med. J. Aust., 1, 46-49

Williams, A. W., Edwards, F., Lewis, T. H. C., and Coghill, N. F. (1957). Investigation on non-ulcer dyspepsia by gastric biopsy. Brit. med. J., 1, 372-377 